\title{
Short pressure reactivity index versus long pressure reactivity index in the management of traumatic brain injury
}

\author{
Erhard W. Lang, MD, PhD, ${ }^{1}$ Magdalena Kasprowicz, PhD, ${ }^{2}$ Peter Smielewski, PhD, ${ }^{3}$ \\ Edgar Santos, MD, ${ }^{4}$ John Pickard, FRCS(SN), FMedSci, ${ }^{3}$ and Marek Czosnyka, $\mathrm{PhD}^{3}$
}

\begin{abstract}
${ }^{1}$ Neurosurgical Associates, Red Cross Hospital, Kassel; ${ }^{4}$ Department of Neurosurgery, University of Heidelberg, Germany; ${ }^{2}$ Institute of Biomedical Engineering and Instrumentation, Wroclaw University of Technology, Wroclaw, Poland; and ${ }^{3}$ Department of Neurosurgery, Addenbrooke's Hospital, University of Cambridge, United Kingdom
\end{abstract}

\begin{abstract}
OBJECT The pressure reactivity index (PRx) correlates with outcome after traumatic brain injury (TBI) and is used to calculate optimal cerebral perfusion pressure (CPPopt). The PRx is a correlation coefficient between slow, spontaneous changes $(0.003-0.05 \mathrm{~Hz})$ in intracranial pressure (ICP) and arterial blood pressure (ABP). A novel index-the so-called long PRx (L-PRx) - that considers ABP and ICP changes $(0.0008-0.008 \mathrm{~Hz})$ was proposed.
\end{abstract}

METHODS The authors compared PRx and L-PRx for 6-month outcome prediction and CPPopt calculation in 307 patients with TBI. The PRx- and L-PRx-based CPPopt were determined and the predictive power and discriminant abilities were compared.

RESULTS The PRx and L-PRx correlation was good ( $R=0.7, p<0.00001$; Spearman test). The PRx, age, CPP, and Glasgow Coma Scale score but not L-PRx were significant fatal outcome predictors (death and persistent vegetative state). There was a significant difference between the areas under the receiver operating characteristic curves calculated for PRx and L-PRx (0.61 \pm 0.04 vs $0.51 \pm 0.04$; z-statistic $=-3.26, p=0.011)$, which indicates a better ability by PRx than L-PRx to predict fatal outcome. The CPPopt was higher for L-PRx than for PRx, without a statistical difference (median CPPopt for L-PRx: $76.9 \mathrm{~mm} \mathrm{Hg}$, interquartile range [IQR] $\pm 10.1 \mathrm{~mm} \mathrm{Hg}$; median CPPopt for PRx: $74.7 \mathrm{~mm} \mathrm{Hg}$, IQR $\pm 8.2 \mathrm{~mm} \mathrm{Hg}$ ). Death was associated with CPP below CPPopt for PRx $\left(\chi^{2}=30.6, p<0.00001\right)$, and severe disability was associated with CPP above CPPopt for $\operatorname{PRx}\left(\chi^{2}=7.8, p=0.005\right)$. These relationships were not statistically significant for CPPopt for L-PRx.

CONCLUSIONS The PRx is superior to the L-PRx for TBI outcome prediction. Individual CPPopt for L-PRx and PRx are not statistically different. Deviations between CPP and CPPopt for PRx are relevant for outcome prediction; those between CPP and CPPopt for L-PRx are not. The PRx uses the entire B-wave spectrum for index calculation, whereas the L-PRX covers only one-third of it. This may explain the performance discrepancy.

http://thejns.org/doi/abs/10.3171/2014.10.JNS14602

KEY WORDS brain monitoring; cerebral autoregulation; cerebrovascular reactivity; severe head injury; outcome; cerebral perfusion pressure; traumatic brain injury

A RTERIAL blood pressure (ABP), intracranial pressure (ICP), cerebral blood flow velocity measured with transcranial Doppler ultrasonography, and cerebral microcirculation assessed by laser Doppler flow are periodical biological signals that can be continuously monitored and recorded in both routine and ICU settings by using invasive and noninvasive techniques and devices. ${ }^{5,12,16,30,32}$
These biological signals will show frequency peaks within certain frequency bands. ${ }^{6,11}$ They can be categorized into two groups: 1) the B waves that occur at approximately $0.5-3$ cycles per minute, which corresponds to a frequency of $0.008-0.05 \mathrm{~Hz} ;, 5,16,23$ and 2) $\mathrm{M}$ waves, which occur at approximately 4-9 cycles per minute, which corresponds to a frequency of $0.07-0.15 \mathrm{~Hz}^{7,12,22}$ The 6 venti-

ABBREVIATIONS ABP = arterial blood pressure; CPPopt = optimal cerebral perfusion pressure; GCS = Glasgow Coma Scale; GOS = Glasgow Outcome Scale; ICP = intracranial pressure; IQR = interquartile range; L-PRx = long pressure reactivity index; $P V S$ = persistent vegetative state; TBI = traumatic brain injury.

SUBMITTED March 16, 2014. ACCEPTED October 21, 2014.

INCLUDE WHEN CITING Published online November 28, 2014; DOI: 10.3171/2014.10.JNS14602.

DISCLOSURE The software for brain monitoring (ICM+) is licensed by the University of Cambridge (Cambridge Enterprise), and Drs. Smielewski and Czosnyka have financial interests in a part of the licensing fee. Dr. Czosnyka is a consultant for Codman J \& J. Dr. Lang is a consultant for GMS Integra. Dr. Kasprowicz is the recipient of a scholarship funded by the Polish Ministry of Science and Higher Education. 
latory cycles per minute ventilation test is a clinical tool to create an artificial stimulus for M-wave activity. ${ }^{8,19}$

All clinical tests of cerebrovascular reactivity require a stimulus variable and a response variable. , $3,10,15,32^{\text {The stim- }}$ ulus is classically an increase or decrease in ABP, which can be applied via an internal stimulus-i.e., ABP-related $\mathrm{B}$ waves or $\mathrm{M}$ waves; or external-i.e., thigh cuff deflation, transient carotid occlusion, pharmacological blood pressure variations, or changes in ventilator settings. The response to the stimulus can be graded, and indices that grade magnitude and/or time can be calculated. In clinical practice these indices can be used to quantify cerebrovascular reactivity, which contributes to cerebral autoregulation. Intact, partially impaired, or completely disrupted cerebral autoregulation has been reported, and various degrees of impairment have prognostic relevance. ${ }^{1,3,13,17,25,32}$

The pressure reactivity index (PRx), which is an index calculated from a moving correlation coefficient between mean ABP and ICP, has gained wide acceptance in ICU settings, and it has generated a large number of publications. 2,3,14,17,27-29 It is clinically most attractive because it does not require an external stimulus or patient manipulation, and routinely monitored data can be used for clinical research. The PRx has been reported to correlate with outcome after traumatic brain injury (TBI), ${ }^{14,17}$ and it is used for online calculation of the optimal cerebral perfusion pressure (CPPopt). ${ }^{2,29}$

The PRx is calculated from mean ABP and ICP within a frequency range of $0.003-0.05 \mathrm{~Hz}$. Recently a new PRx, called long (L)-PRx, that considers slower changes in ABP and ICP (within a frequency range of $0.0008-0.008 \mathrm{~Hz}$ ) has been introduced. ${ }^{27,28}$ Its utility and prognostic relevance have been confirmed in a small series of 18 patients with spontaneous intracranial hemorrhage, ${ }^{28}$ and in a second series of 29 patients with TBI. ${ }^{27}$

The aim of our study was to investigate, by reference to PRx performance, the utility and performance of the L-PRx for long-term outcome assessment and CPPopt calculation in a large TBI series.

\section{Methods}

We retrospectively analyzed digital recordings of ABP and ICP waveforms from 307 patients with TBI who were hospitalized and treated at the Neurocritical Care Unit at Addenbrooke's Hospital, Cambridge University, United Kingdom, between 2003 and 2009 with the ICM+ software (http://www.neurosurg.cam.ac.uk/icmplus). The outcome assessed by the Glasgow Outcome Scale (GOS) at 6-month follow-up was available for 302 patients.

The PRx and L-PRx values were calculated as the moving linear correlation coefficients between 30 samples of time-averaged (10-second period) data points of ICP and ABP (PRx) and 20 samples of time-averaged (60-second period) data points of ICP and ABP (L-PRx; Fig. 1).

A curve-fitting method was applied to determine the CPPopt for PRx and that for L-PRx in an individual patient, as the CPP associated with the minimum value of PRx and L-PRx, respectively, when plotted against CPP (Fig. 2). In individual patients, the difference between either CPPopt for PRx or CPPopt for L-PRx and mean CPP for the whole monitoring period was calculated. Then the chi-square test was used to compare the rate of the positive (CPP - CPPopt $>0$ ) and negative differences (CPP - CPPopt <0) in groups of patients with severe disability and persistent vegetative state (PVS), and those who died.

Logistic regression was used to examine the association between either PRx or L-PRx and fatal outcome, which we herein define as PVS and death, with adjustment for age, CPP, and admission Glasgow Coma Scale (GCS) score. The areas under the receiver operating characteristic curves were used to compare the discriminant abilities and predictive power of both indices.

The nonparametric Mann-Whitney U-test was used to compare between the dichotomized outcome groups fatal (PVS and death) versus nonfatal (good, moderate, and severe disability) for both PRx and L-PRx. The level of significance was set at 0.05 .

Computerized data monitoring that supports critical care management is standard clinical practice on the Neurosurgical ICU at Addenbrooke's Hospital. Use of the data for anonymous audit and publications is approved by the local ethics committee.

\section{Results}

Table 1 provides an overview of patients' demographic data, clinical variables, and outcomes. The overall median age was $36 \pm 26$ years (median \pm interquartile range [IQR]), and 233 of the 302 patients were male (77\%). There was an overall good correlation between averaged values of PRx and L-PRx ( $\mathrm{R}=0.70, \mathrm{p}<0.00001$; Spearman test $)$.

The PRx, age, CPP, and GCS score but not the L-PRx were significant predictors of fatal outcome (PVS and death), based on the Wald criterion $(p<0.05)$. A test of the full model against the constant-only model was statistically significant, indicating that the set of predictors of the PRx $(\mathrm{p}=0.046)$, age $(\mathrm{p}<0.000001), \mathrm{CPP}(\mathrm{p}<0.002)$, and GCS score $(p<0.002)$ reliably distinguished between $p a-$ tients with fatal outcome $(\mathrm{GOS} \geq 4)$ and nonfatal outcome $(\mathrm{GOS}<4)$ at 6 months after discharge from hospital $\left(\chi^{2}\right.$ $=46.9, \mathrm{p}<0.000001$ with $\mathrm{df}=4)$. The logistic regression model is described by the equation:

$$
\mathrm{P} 1(\mathrm{X})=\frac{\mathrm{e}^{3.08-0.15 * \mathrm{GCS}+0.04 * \mathrm{Age}-0.07 * \mathrm{CPP}+1.98 \mathrm{PRx}}}{1+\mathrm{e}^{3.08-0.15^{*} \mathrm{GCS}+0.04 * \mathrm{Age}-0.07 * \mathrm{CPP}+1.98 \mathrm{PRx}}}
$$

The classification performance values for discrimination between fatal and nonfatal outcome were as follows: good accuracy $(78.3 \%)$ and excellent sensitivity $(95.9 \%)$, but poor specificity (25.7\%). An analysis of the odds ratios (Table 2) showed that of all the predictors an increase in the PRx most significantly increases the chance of fatal outcome. Nevertheless, wide $95 \%$ confidence limits of the odds ratio for PRx might suggest low precision of this estimation.

There was a significant difference between areas under the receiver operating characteristic curves calculated for the PRx and the L-PRx $(0.61 \pm 0.04$ vs $0.51 \pm 0.04$; $\mathrm{z}$ statistic $=-3.26, p=0.011)$. It suggests better ability of PRx than L-PRx to predict fatal outcome (PVS and death; Fig. 3). 
E. W. Lang et al.

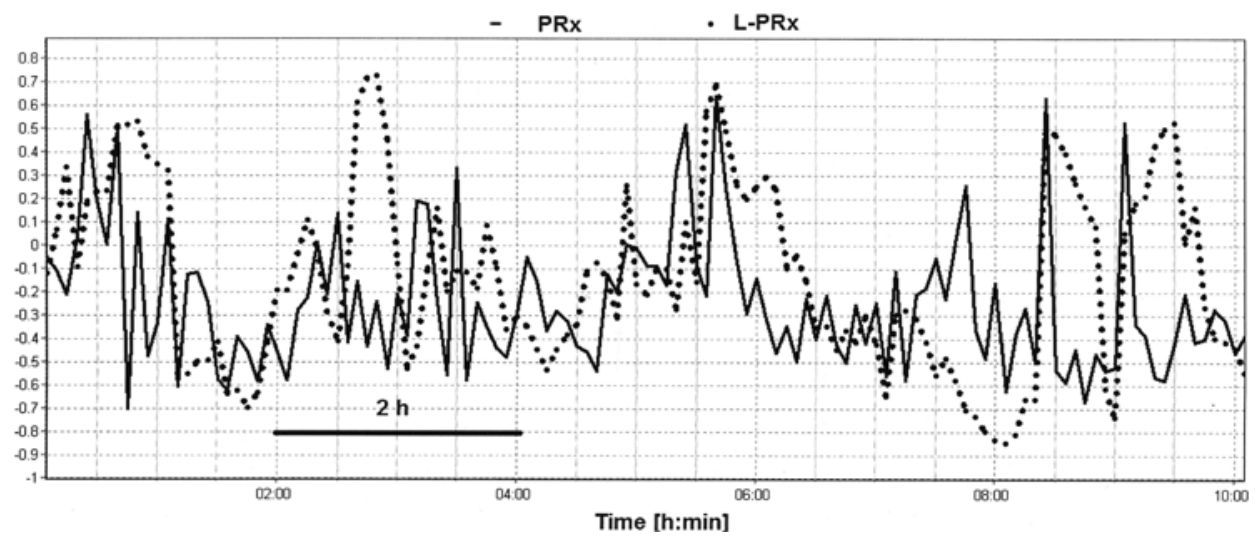

FIG. 1. Chart showing an example of the PRx and L-PRx values (y axis) recorded over a 10-hour-period. Note that although the trending appears to follow a common pattern, there are several instances where PRx and L-PRx differ considerably.

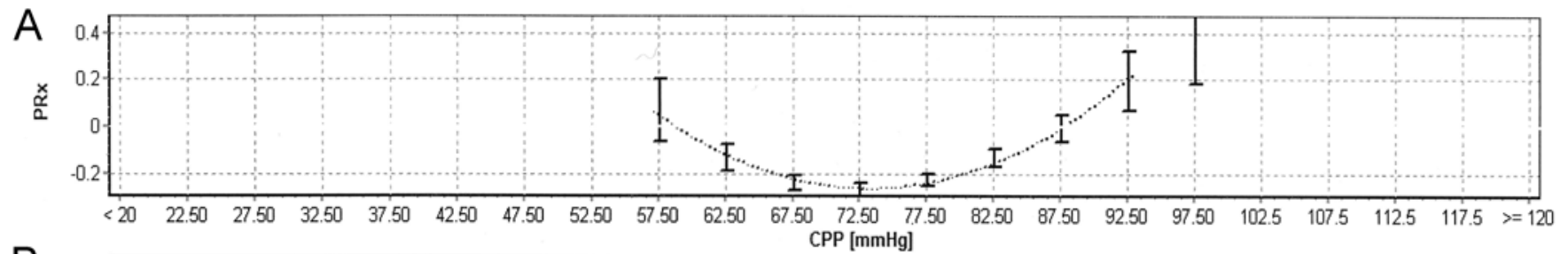

B

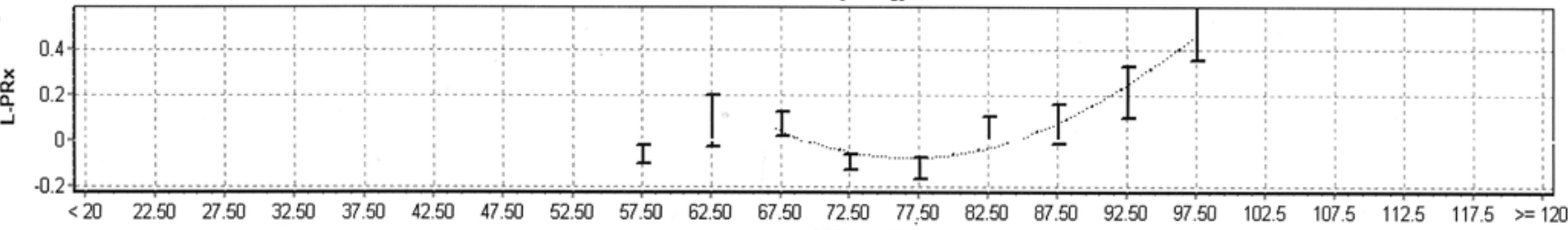

C

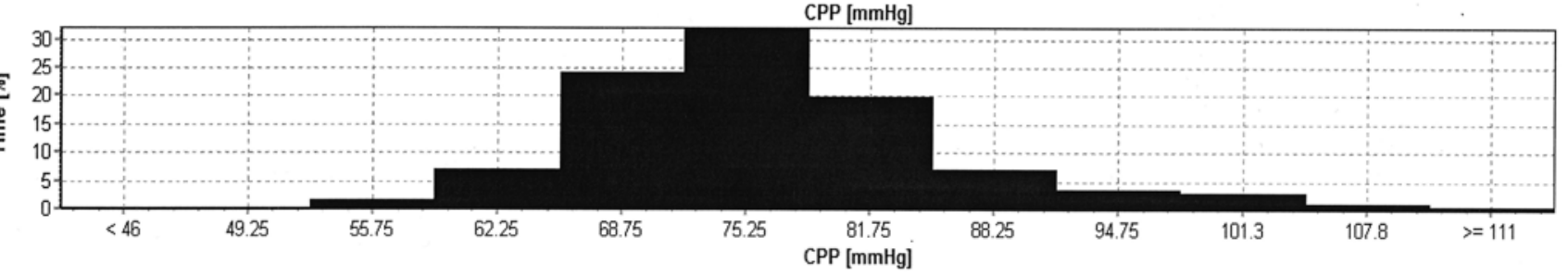

FIG. 2. Plots showing PRx (A) and L-PRx (B) examples versus CPP plotted in an individual patient. The CPPopt values are 72.5 $\mathrm{mm} \mathrm{Hg}$ for CPPopt-PRx (A) and $77.5 \mathrm{~mm} \mathrm{Hg}$ for CPPopt-L-PRx (B). Panel $\mathbf{C}$ shows the percentage of time spent within individual CPP intervals.

TABLE 1. Demographic data, clinical variables, and outcome at 6 months in 302 patients with TBI*

\begin{tabular}{lrcccccccc}
\hline Outcome & Total No. & No. Male & Age $(\mathrm{yrs})$ & GCS Score & ICP & ABP & CPP & PRx & L-PRx \\
\hline Good & 48 & 30 & $27 \pm 24$ & $7 \pm 4.5$ & $16.6 \pm 6.4$ & $94.7 \pm 6.5$ & $77.1 \pm 6.4$ & $0.035 \pm 0.203$ & $0.020 \pm 0.176$ \\
\hline Moderate & 79 & 66 & $29 \pm 19$ & $8 \pm 6$ & $15.7 \pm 4.9$ & $93.8 \pm 7.8$ & $77.8 \pm 6.0$ & $0.064 \pm 0.193$ & $0.038 \pm 0.198$ \\
\hline Severe & 100 & 75 & $38 \pm 24$ & $5 \pm 5$ & $16.0 \pm 5.6$ & $94.6 \pm 9.1$ & $78.4 \pm 9.2$ & $0.070 \pm 0.178$ & $0.050 \pm 0.267$ \\
\hline PVS & 9 & 7 & $38 \pm 26$ & $5 \pm 5$ & $15.5 \pm 4.5$ & $86.1 \pm 11.1$ & $74.2 \pm 9.3$ & $0.018 \pm 0.044$ & $0.033 \pm 0.216$ \\
\hline Dead & 66 & 55 & $45 \pm 31$ & $5 \pm 5$ & $18.8 \pm 9.0$ & $96.1 \pm 9.5$ & $75.3 \pm 11.5$ & $0.140 \pm 0.186$ & $0.042 \pm 0.237$ \\
\hline
\end{tabular}

* Unless otherwise noted, values are the median \pm IQR. Units of measure for the ICP, ABP, and CPP: mm Hg; PRx and L-PRx are the correlation coefficients. 
TABLE 2. Comparison of predictors of fatal outcome in patients with TBI

\begin{tabular}{lcc}
\hline Variable & Unadjusted OR $(95 \% \mathrm{Cl})$ & Adjusted OR $(95 \% \mathrm{Cl})$ \\
\hline GCS score & $0.90(0.83-0.99)$ & $0.86(0.78-0.94)$ \\
\hline Age & $1.04(1.02-1.05)$ & $1.04(1.03-1.07)$ \\
\hline CPP & $0.94(0.91-0.98)$ & $0.94(0.90-0.98)$ \\
\hline PRx & $19.7(3.18-122)$ & $7.24(1.03-50.7)$ \\
\hline L-PRx & NS & NS \\
\hline
\end{tabular}

NS = not significant.

The CPPopt values for pooled data were higher by approximately $5 \mathrm{~mm} \mathrm{Hg}$ for L-PRx than for PRx (Fig. 4). Individual CPPopt for PRx was identified in 299 of 307 patients (97.4\%), and that for L-PRx was found in 300 patients. There was no statistical difference between CPPopt for PRx and L-PRx (median $74.7 \mathrm{~mm} \mathrm{Hg}, \mathrm{IQR} \pm 8.2 \mathrm{~mm}$ $\mathrm{Hg}$ vs median $76.9 \mathrm{~mm} \mathrm{Hg}, \mathrm{IQR} \pm 10.1 \mathrm{~mm} \mathrm{Hg}$ ).

Mortality was associated with a mean CPP below the CPPopt for PRx $\left(\chi^{2}=30.6, \mathrm{p}<0.00001\right)$, whereas severe disability was associated with CPP above the CPPopt for $\operatorname{PRx}\left(\chi^{2}=7.8, p=0.005\right)$. These relationships were not statistically significant for CPPopt calculated for L-PRx (Fig. 5).

\section{Discussion}

This study investigates the utility of two indices of cerebrovascular reactivity for clinical practice in patients with TBI, and it shows that both PRx and L-PRx can be calculated from monitored variables in almost all patients. Calculated from different frequencies, they correlate well, although in our particular series the PRx is superior to LPRx for TBI outcome prediction.

In their first comparative study Santos et al. report a PRx-L-PRx correlation of $\mathrm{R}=0.84,{ }^{28}$ which is higher than in our study $(\mathrm{R}=0.7)$. It must be noted, however, that their series consists of 18 patients with spontaneous intracerebral hemorrhage, which may represent a less variable clinical entity than our series of 307 patients with TBI. These numerical and etiological differences are likely to explain this slight discrepancy.

Interestingly, they also report that: "L-PRx-CPPopt was found to be the same as PRx-CPPopt in all but one patient" and recommend a larger series for confirmation of their findings. ${ }^{28}$ This larger series, which we present herein, now shows an approximately $5 \mathrm{~mm} \mathrm{Hg}$ difference between the two indices for CPPopt calculation, which is not statistically significant, and on top of that may represent a number that we consider difficult to target and/or maintain in a clinical ICU setting.

In their second series of 29 patients with TBI they report a significant L-PRx difference between survival and death. ${ }^{27}$ Our large TBI series shows that the PRx is significantly higher in patients with fatal outcome (PVS and death) than in patients with nonfatal outcome (good, moderate, severe disability), which we cannot confirm for the L-PRx. This finding brings us to a previously raised issue. This particular study as well as the cited ones are not meant to enter a competition in search of the most superior outcome prediction tool or technique. We wish to stress the point that we assess the applicability and performance of indices of cerebrovascular reactivity on a given data set, for which we confirm or refute its utility.

By now researchers have identified numerous univariate-analysis patient- and treatment-associated factors that contribute to outcome, such as age, GCS and GCS subscores, injury patterns, sex, time of data acquisition, CPP, ICP, cerebral blood flow, cerebral partial pressure of oxygen, coagulation status, and so on. . $13,14,18,20,21,24,26^{\text {The }}$ specific composition of any clinical data set is therefore highly variable. This points toward the complexity of TBI, and it becomes highly difficult if not impossible to control or correct for any one of these confounding variables in outcome prediction. This answers the question why different indices perform differently in different data sets.

Based on previously published data from the Cambridge group, which were derived from a different data set, the CPPopt concept has been suggested. ${ }^{29}$ Steiner et al. as well as Aries et al. showed that an increasing $\Delta \mathrm{CPP} / \mathrm{CPPopt}$ correlated with less favorable outcome after TBI. ${ }^{2,29}$ Our study confirms the concept and utility of CPPopt. It also shows that although individual CPPopt for L-PRx and PRx were not statistically different, deviations between CPP and CPPopt for PRx are relevant for outcome prediction, whereas those between CPP and CPPopt for LPRx are not. This once again raises the issue of the clinical utility of the indices, and it shows that the PRx offers an opportunity that the L-PRx does not.
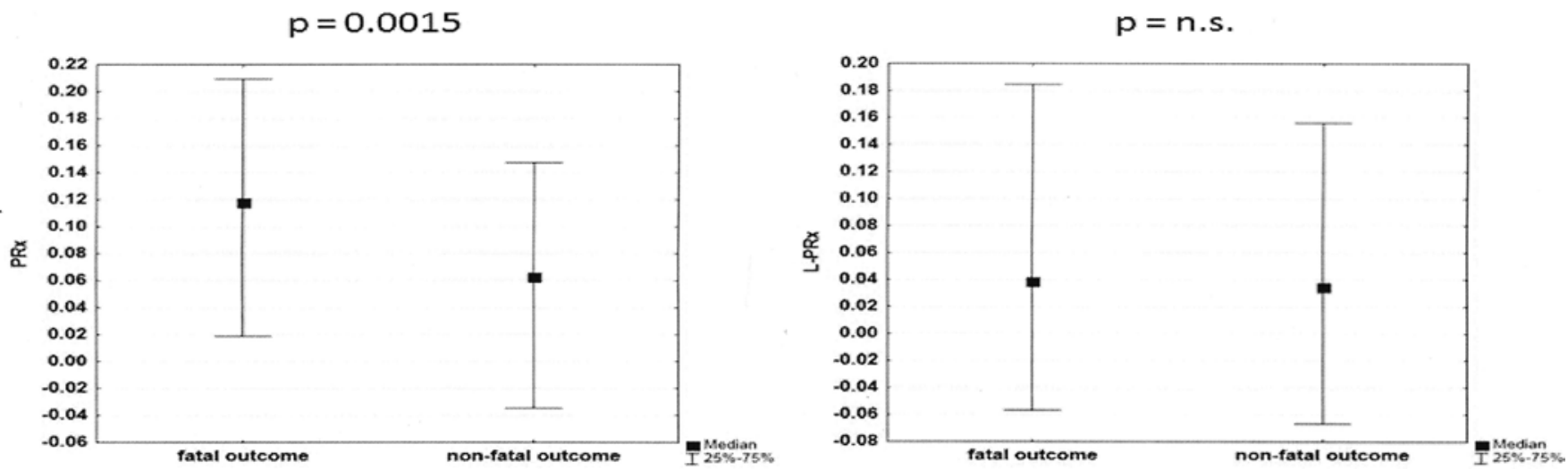

FIG. 3. Plots showing better ability of PRx than L-PRx to predict fatal outcome (PVS and death). n.s. = not significant. 

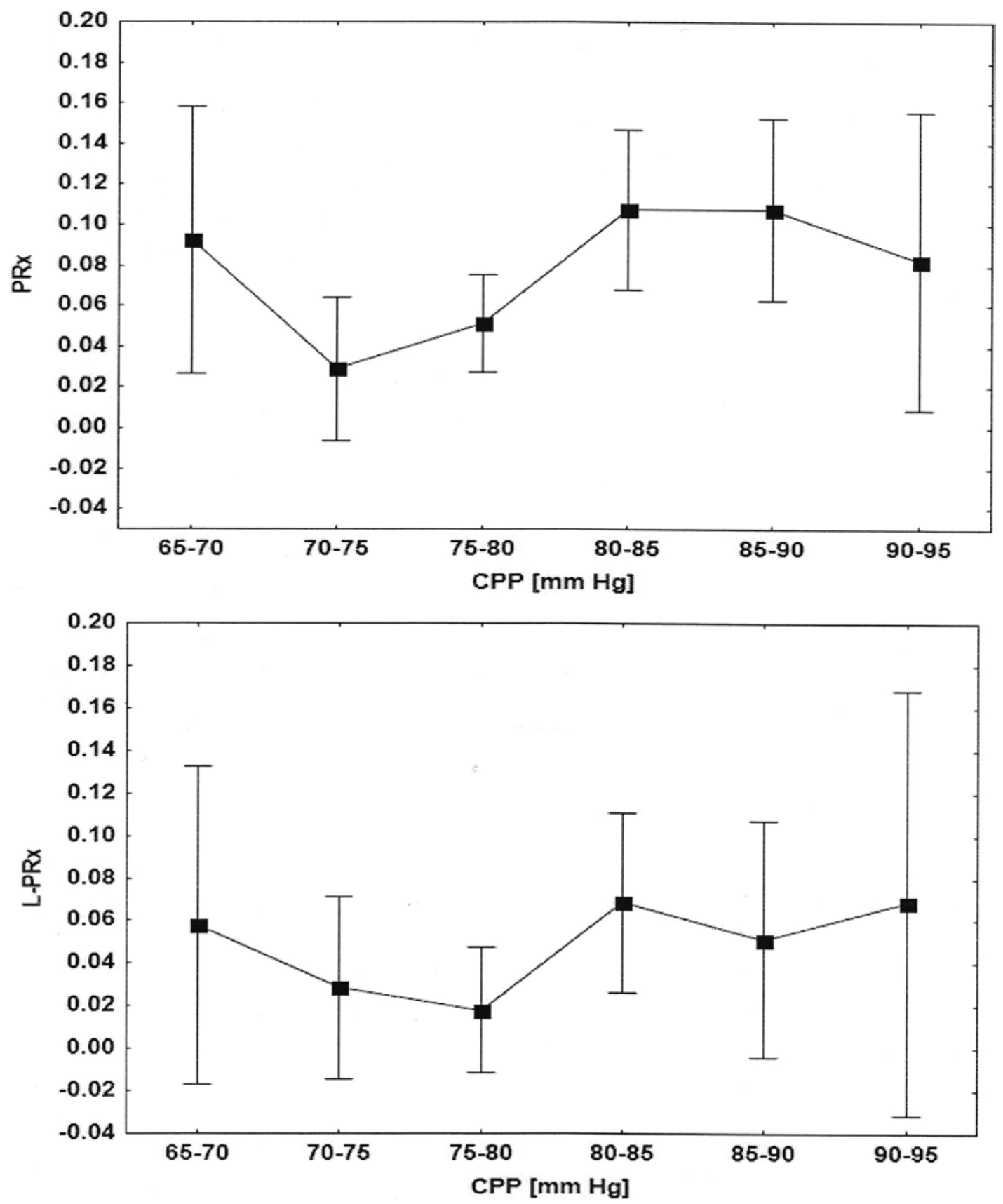

FIG. 4. Plots showing PRx and L-PRx versus CPP for pooled data from all 307 patients. The CPPopt for PRx (upper plot) was demonstrated at $70-75 \mathrm{~mm} \mathrm{Hg}$, whereas CPPopt for L-PRx (lower plot) was at $75-80 \mathrm{~mm} \mathrm{Hg}$.

In the search for an explanation of this discrepancy it becomes important to consider the physiological basis referred to in the introduction. The PRx is calculated from the frequency that represents the entire B-wave activity: 0.5-3 cycles per minute. The cycles represent the body's intrinsic generators and a sufficient activity per cycle must be generated to evoke and calculate autoregulatory responses. The L-PRx calculation only covers the spectrum up to 1 cycle per minute (slow frequency components within the 0.5-3 cycles per minute waveband). This translates into the fact that the L-PRx uses only one-third of its potential input activity and is likely to lack essential autoregulatory-gen- erating elements with limited cerebrovascular reactivity representation. This is likely to be the cause for the L-PRx lack of performance in this particular matter.

We also report that death is associated with a mean CPP below CPPopt for the PRx. Interestingly, severe disability is associated with a mean CPP above CPPopt for the PRx. This has been reported in an analysis of the same database at Addenbrooke's Hospital. ${ }^{2}$ In a rather simple clinical interpretation, this indicates that too-high CPP produces unfavorable outcomes and that too-low CPP causes death. More detailed considerations and the discussion about this issue can be found in the original paper. ${ }^{2}$ 

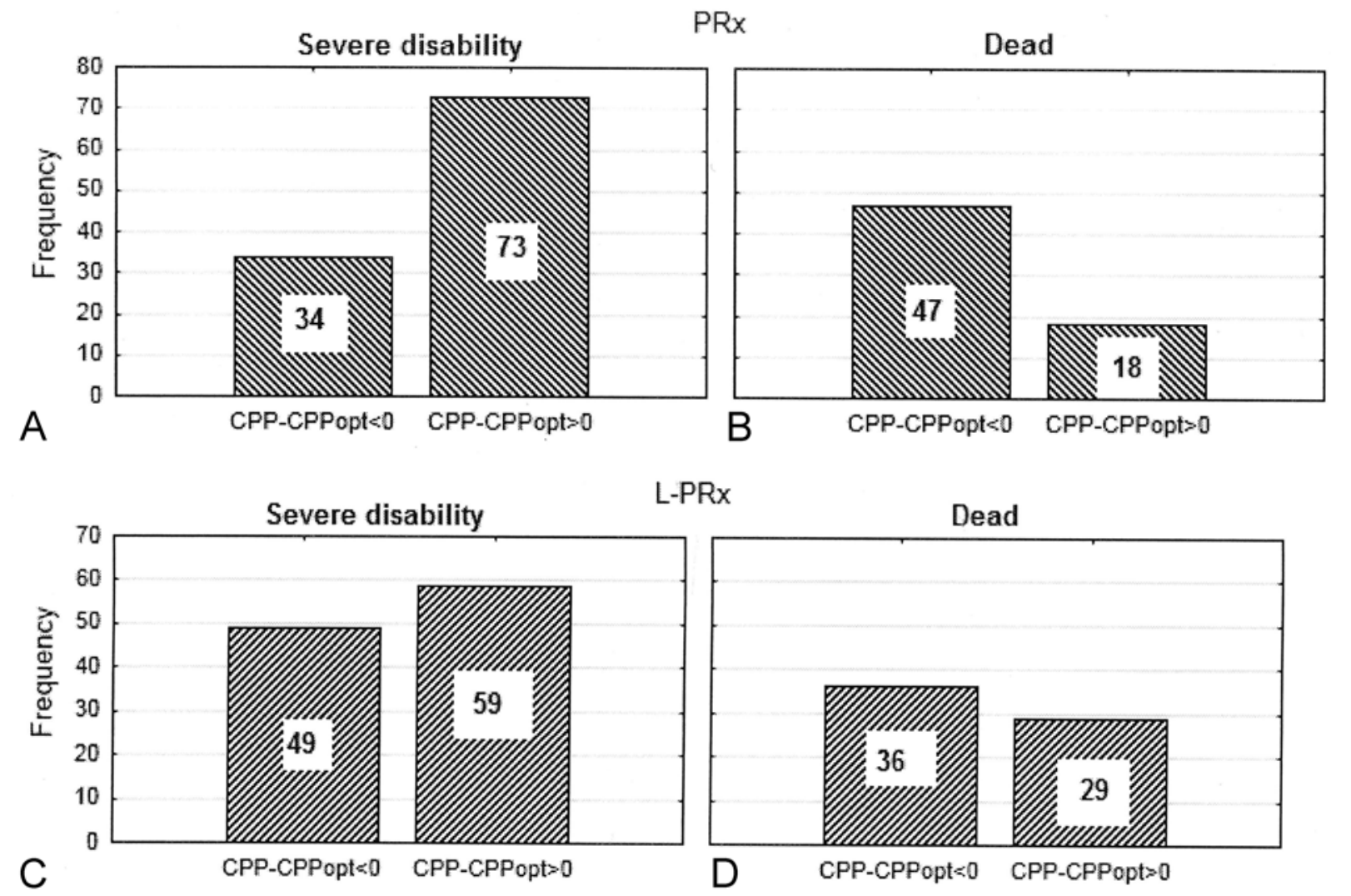

FIG. 5. Bar graphs showing the relationship between severe disability rate (A and $C)$ and mortality rate (B and $D)$ for the difference between mean CPP and CPPopt calculated for the PRx (A and B) and the L-PRx (C and D). See text for values. Totals are slightly lower in this figure because data were not available in some patients.

A recent paper has taken this issue further, and it also points toward the limitations of our analysis..$^{14}$ Johnson et al. dichotomized 107 patients with TBI, in keeping with the Marshall classification, ${ }^{21}$ into "diffuse" (Marshall Types I, II, and III) and "focal” (Marshall Types diffuseIV, evacuated mass lesion, and nonevacuated mass lesion) groups. They confirmed that a low PRx was significantly associated with favorable outcome for the combined and the diffuse groups. This was not confirmed for the focal group..$^{14}$ This points toward the fact that the utility of any global index may not pick up the heterogeneity of various types of head injury patterns. This calls for a further analysis of TBI subsets to validate the utility and performance of indices of cerebrovascular reactivity for long-term outcome assessment and CPPopt calculation.

Depreitere and colleagues presented a new index called the Low-frequency Autoregulation index (LAx), where they also use minute-by-minute data of ICP and mean ABP in patients with head trauma (as in L-PRx). ${ }^{4}$ Their intention was to assess the vascular pressure reactivity of cerebral autoregulation with an algorithm called the Dynamic Adaptive Target of Active Cerebral AutoRegulation (DATACAR). This methodology has been tested using historical data up to 24 hours preceding the time point of interest, and allowed the identification of CPPopt with a high success rate. The time that the subjects were close to this individualized CPPopt correlated with the outcome, although for unknown reasons the authors used only the first 48 hours of monitoring information. In our opinion, CPPopt should be used to guide the whole period of intensive management, particularly through periods of secondary intracranial hypertension. ${ }^{31}$

\section{Conclusions}

The PRx allows more precise outcome prediction than the L-PRx. Deviations from CPPopt obtained for PRx were more predictive than those calculated for L-PRx, although the individual values of CPPopt for L-PRx and PRx were not statistically different. The PRx uses the entire B-wave spectrum for index calculation, whereas the L-PRx covers only one-third of it. This may explain the performance discrepancy.

\section{Acknowledgments}

All authors thank the Neurocritical Care Unit staff members at Addenbrooke's Hospital for their active involvement and support during the study.

\section{References}

1. Aaslid R, Newell DW, Stooss R, Sorteberg W, Lindegaard KF: Assessment of cerebral autoregulation dynamics from simultaneous arterial and venous transcranial Doppler recordings in humans. Stroke 22:1148-1154, 1991

2. Aries MJ, Czosnyka M, Budohoski KP, Steiner LA, Lavinio A, Kolias AG, et al: Continuous determination of optimal cerebral perfusion pressure in traumatic brain injury. Crit Care Med 40:2456-2463, 2012 
3. Czosnyka M, Smielewski P, Kirkpatrick P, Laing RJ, Menon D, Pickard JD: Continuous assessment of the cerebral vasomotor reactivity in head injury. Neurosurgery 41:11-19, 1997

4. Depreitere B, Güiza F, Van den Berghe G, Schuhmann MU, Maier G, Piper I, et al: Pressure autoregulation monitoring and cerebral perfusion pressure target recommendation in patients with severe traumatic brain injury based on minute-by-minute monitoring data. J Neurosurg 120:1451-1457, 2014

5. Diehl RR: B-waves and cerebral autoregulation. Stroke 28:1289-1291, 1997

6. Diehl RR: Funktionelle Dopplersonographie in der Neurologie. Berlin: Springer, 1996

7. Diehl RR, Diehl B, Sitzer M, Hennerici M: Spontaneous oscillations in cerebral blood flow velocity in normal humans and in patients with carotid artery disease. Neurosci Lett 127:5-8, 1991

8. Diehl RR, Linden D, Lücke D, Berlit P: Phase relationship between cerebral blood flow velocity and blood pressure. A clinical test of autoregulation. Stroke 26:1801-1804, 1995

9. Firsching R, Woischneck D, Klein S, Reissberg S, Döhring W, Peters B: Classification of severe head injury based on magnetic resonance imaging. Acta Neurochir (Wien) 143:263-271, 2001

10. Giller CA: A bedside test for cerebral autoregulation using transcranial Doppler ultrasound. Acta Neurochir (Wien) 108:7-14, 1991

11. Giller CA: The frequency-dependent behavior of cerebral autoregulation. Neurosurgery 27:362-368, 1990

12. Haubrich C, Klemm A, Diehl RR, Möller-Hartmann W, Klötzsch C: M-wave analysis and passive tilt in patients with different degrees of carotid artery disease. Acta Neurol Scand 109:210-216, 2004

13. Jaeger M, Soehle M, Schuhmann MU, Meixensberger J: Clinical significance of impaired cerebrovascular autoregulation after severe aneurysmal subarachnoid hemorrhage. Stroke 43:2097-2101, 2012

14. Johnson U, Lewén A, Ronne-Engström E, Howells T, Enblad $\mathrm{P}$ : Should the neurointensive care management of traumatic brain injury patients be individualized according to autoregulation status and injury subtype? Neurocrit Care 21:259-265, 2014

15. Lang EW, Czosnyka M, Mehdorn HM: Tissue oxygen reactivity and cerebral autoregulation after severe traumatic brain injury. Crit Care Med 31:267-271, 2003

16. Lang EW, Diehl RR, Timmermann L, Baron R, Deuschl G, Mehdorn HM, et al: Spontaneous oscillations of arterial blood pressure, cerebral and peripheral blood flow in healthy and comatose subjects. Neurol Res 21:665-669, 1999

17. Lang EW, Lagopoulos J, Griffith J, Yip K, Mudaliar Y, Mehdorn HM, et al: Noninvasive cerebrovascular autoregulation assessment in traumatic brain injury: validation and utility. $\mathbf{J}$ Neurotrauma 20:69-75, 2003

18. Lang EW, Pitts LH, Damron SL, Rutledge R: Outcome after severe head injury: an analysis of prediction based upon comparison of neural network versus logistic regression analysis. Neurol Res 19:274-280, 1997

19. Lewis PM, Rosenfeld JV, Diehl RR, Mehdorn HM, Lang EW: Phase shift and correlation coefficient measurement of cerebral autoregulation during deep breathing in traumatic brain injury (TBI). Acta Neurochir (Wien) 150:139-147, 2008

20. Marion DW, Darby J, Yonas H: Acute regional cerebral blood flow changes caused by severe head injuries. J Neurosurg 74:407-414, 1991

21. Marshall LF, Marshall SB, Klauber MR, Van Berkum Clark M, Eisenberg H, Jane JA, et al: The diagnosis of head injury requires a classification based on computed axial tomography. J Neurotrauma 9 (Suppl 1):S287-S292, 1992

22. Mayer S: Studien zur Physiologie des Herzens und der Blutgefässe. Sitz Kaiser Akad Wiss 74:281-307, 1876
23. Newell DW, Aaslid R, Stooss R, Reulen HJ: The relationship of blood flow velocity fluctuations to intracranial pressure B waves. J Neurosurg 76:415-421, 1992

24. Oddo M, Levine JM, Mackenzie L, Frangos S, Feihl F, Kasner $\mathrm{SE}$, et al: Brain hypoxia is associated with short-term outcome after severe traumatic brain injury independently of intracranial hypertension and low cerebral perfusion pressure. Neurosurgery 69: 1037-1045, 2011

25. Oehm E, Hetzel A, Els T, Berlis A, Keck C, Will HG, et al: Cerebral hemodynamics and autoregulation in reversible posterior leukoencephalopathy syndrome caused by pre-/eclampsia. Cerebrovasc Dis 22:204-208, 2006

26. Pieracci FM, Eachempati SR, Shou J, Hydo LJ, Barie PS: Degree of anticoagulation, but not warfarin use itself, predicts adverse outcomes after traumatic brain injury in elderly trauma patients. J Trauma 63:525-530, 2007

27. Sánchez-Porras R, Santos E, Czosnyka M, Zheng Z, Unterberg AW, Sakowitz OW: 'Long' pressure reactivity index (L-PRx) as a measure of autoregulation correlates with outcome in traumatic brain injury patients. Acta Neurochir (Wien) 154:15751581,2012

28. Santos E, Diedler J, Sykora M, Orakcioglu B, Kentar M, Czosnyka M, et al: Low-frequency sampling for PRx calculation does not reduce prognostication and produces similar CPPopt in intracerebral haemorrhage patients. Acta Neurochir (Wien) 153:2189-2195, 2011

29. Steiner LA, Czosnyka M, Piechnik SK, Smielewski P, Chatfield D, Menon DK, et al: Continuous monitoring of cerebrovascular pressure reactivity allows determination of optimal cerebral perfusion pressure in patients with traumatic brain injury. Crit Care Med 30:733-738, 2002

30. Steinmeier R, Bauhuf C, Hübner U, Bauer RD, Fahlbusch R, Laumer R, et al: Slow rhythmic oscillations of blood pressure, intracranial pressure, microcirculation, and cerebral oxygenation. Dynamic interrelation and time course in humans. Stroke 27:2236-2243, 1996

31. Strege RJ, Lang EW, Stark AM, Scheffner H, Fritsch MJ, Barth H, et al: Cerebral edema leading to decompressive craniectomy: an assessment of the preceding clinical and neuromonitoring trends. Neurol Res 25:510-515, 2003

32. Tiecks FP, Lam AM, Aaslid R, Newell DW: Comparison of static and dynamic cerebral autoregulation measurements. Stroke 26:1014-1019, 1995

\section{Author Contributions}

Conception and design: Smielewski, Santos, Pickard, Czosnyka. Acquisition of data: Smielewski, Pickard, Czosnyka. Analysis and interpretation of data: Kasprowicz, Smielewski, Santos, Czosnyka, Lang. Drafting the article: Lang. Critically revising the article: Lang, Kasprowicz, Smielewski, Czosnyka. Reviewed submitted version of manuscript: Lang, Santos. Approved the final version of the manuscript on behalf of all authors: Lang. Statistical analysis: Kasprowicz, Smielewski, Czosnyka. Administrative/technical/material support: Pickard. Study supervision: Pickard, Czosnyka.

\section{Supplemental Information}

Previous Presentation

This work was presented at the 15th International Conference on Intracranial Pressure and Brain Monitoring, which was held in Singapore, November 6-10, 2013.

\section{Correspondence}

Erhard W. Lang, Neurosurgery Associates, Red Cross Hospital, Bergmannstrasse 30, D-34121 Kassel, Germany. email: keeflang@online.de. 